\section{Vasoprotection as neuroprotection for the optic nerve}

Neuroprotective measures may in the future be able to slow the cellular damage and cell death within the eye that characterises glaucoma, anterior ischaemic optic neuropathy (AION) and age-related macular degeneration (AMD). ${ }^{1}$ However, preventing cell injury by preserving adequate nutrient delivery - so-called vasoprotection - may also help manage these diseases.

Ischaemia, hypoxia and hypoglycaemia reduce oxygen or glucose delivery to neural tissue. Maintaining adequate nutrient supply consequently provides 'vasoprotective' effects. The relationship of vasoprotection to neuroprotection exists on several levels. First, in cell culture, hypoxia, ischaemia or anoxia are routinely used to accelerate neuronal death, allowing cellular injury and potential neuroprotective interventions to be studied. Second, within the brain itself, ischaemia (and subsequent reperfusion) induced by stroke or cardiac insufficiency is a major cause of morbidity and mortality. ${ }^{2}$ Clearly, in this instance, vasoprotection has failed, and development of novel neuroprotective techniques is essential for progress. Third, emerging evidence finds that experimentally imposed ischaemic insults can generate the specific biochemical and histological changes seen in Alzheimer's disease. These results taken together suggest that the cellular pathological response to vascular insufficiency can be extraordinarily diverse. Finally, while the role that ischaemia may play in glaucoma, AION and AMD remains unresolved, at least a subset of these patients may suffer from a primary ischaemic insult. ${ }^{3}$

In cell culture, hypoxia and glutamate (an excitotoxic agent) each accelerate the death of retinal ganglion cells. ${ }^{4}$ Identical in their effects, these two cell stressors are used to study agents that may decrease cellular susceptibility to necrosis or apoptosis. For example, a noncompetitive $N$-methyl--D,L--aspartate (NMDA) receptor antagonist, MK-801, increases neuronal resistance to hypoxic or glutamate toxicity, as does the presence of Müller glia. ${ }^{4}$ Similarly, a 72 $\mathrm{kDa}$ heat shock protein, synthesised by cells under sublethal stress, increases subsequent cellular resistance to glutamate or hypoxia. ${ }^{5}$ Further, endogenous nitric oxide may be potentially cytotoxic, since nitric oxide synthase inhibitors reduce neuronal susceptibility to either glutamate or hypoxia. ${ }^{6}$ Some of these strategies may eventually, after questions regarding treatment delivery and side effects are resolved, play neuroprotective roles in ocular disease. ${ }^{1,3}$

Ischaemic-reperfusion injury in the brain involves the acute, subacute and chronic cellular responses of neurons, glia and vascular endothelium. ${ }^{7}$ Neuronal loss is associated with a delayed proliferation of reactive microglia, and with reactive astrocytosis. Since stroke represents a failure of vasoprotection, research has focused upon the possibility for neuroprotective mitigation of these cellular pathological responses. For example, because mice that overexpress mitochondrial manganese superoxide dismutase resist CNS neuronal ischaemic cell death, superoxide and peroxynitrite production may represent blockable targets to reduce cellular susceptibility to insult. ${ }^{8}$ In contrast, mice deficient in the interleukin-1-beta converting enzyme show reduced brain injury during ischaemia, suggesting that this regulatory factor for both inflammation and apoptosis may be a target for therapeutic intervention. ${ }^{2}$ Because cells undergoing ischaemic injury express a variety of proteins, identification of these markers in ischaemic retinal ganglion cells, photoreceptors and retinal pigment epithelium could increase understanding of factors mediating cell damage or loss, and provide future targets for intervention, in ocular disease. ${ }^{9}$

While stroke clearly represents a failure of vasoprotection of the brain, there is emerging evidence that experimentally imposed vascular deficiency can cause the specific pathological changes seen in the neurodegenerative dementias such as Alzheimer's disease. For example, presenilin-1 and -2, amyloid precursor protein and apolipoprotein E, all Alzheimer'srelated genes, are induced by ischaemia. ${ }^{10}$ Because cerebral ischaemia and hypoxia stimulate astrogliosis and angiogenesis, clusters of reactive astrocytes expressing increased vascular endothelial growth factor in Alzheimer's disease may arise from ischaemia or oligaemia. ${ }^{11}$ Further, tau dephosphorylation occurs in Alzheimer's disease and after oxygen
A. Harris

T.A. Ciulla

L. Kagemann

D. Zarfati

Department of

Ophthalmology

Indiana University School of Medicine

Indianapolis

Indiana, USA

B. Martin

Medical Sciences Program Indiana University

Bloomington

Indiana, USA

Alon Harris, PhD

Department of

Ophthalmology

Rotary 137A

Indiana University School of Medicine

Indianapolis

IN 46202, USA

Tel: +1 (317) 2782566

Fax: +1 (317) 2781007

e-mail: alharris@indiana.edu

Supported in part by $\mathrm{NIH}$ grant EY10801 (A.H.) 
or glucose deprivation. ${ }^{12}$ One possible mechanism by which oestrogen treatment may prove beneficial to Alzheimer's patients is by reducing overexpression of amyloid precursor protein mRNA after focal ischaemia in the rat brain. ${ }^{13}$

These multiple pathological changes subsequent to simple ischaemic insult reveal the diverse cellular responses to nutrient insufficiency. Pathological changes may be influenced by the specific nutrient deficiency, by the metabolic capacities of the affected cells, and by the severity and duration of the insult. For example, although the acute response to cerebral ischaemia is neuronal necrosis, the prolonged response includes abnormal deposition or beta-amyloid precursor protein. ${ }^{14}$ Beta-amyloid precursor protein can appear in regions devoid of overt ischaemic neuronal damage, suggesting that subclinical nutrient insufficiency can lead to specific neuronal pathologies. ${ }^{14}$

While ischaemia clearly provokes CNS neuronal damage in vitro and in vivo after stroke or cardiac insufficiency, and may play a role in the pathology of Alzheimer's disease, ischaemia as a factor in glaucoma, AION or AMD remains controversial. Although there are unambiguous circulatory deficits in each of these diseases, vascular abnormalities in the retina, choroid, retrobulbar vessels or optic nerve head may be either primary or secondary to non-vascular events. ${ }^{3,15}$ For example, glaucoma patients exhibit delayed choroidal filling, ${ }^{16}$ increased vascular resistance distal to retrobulbar arteries ${ }^{17}$ and reduced retinal capillary perfusion. ${ }^{18}$ The available experimental evidence is insufficient to determine whether these changes predispose to glaucoma or whether they simply reflect vascular adjustments to a more fundamental loss of retinal ganglion cells and photoreceptors. In AMD, vascular resistance within the choroid is elevated, ${ }^{15}$ delaying choroidal filling in proportion to the slowing of the foveal cone electroretinogram. ${ }^{19}$ Again, choriocapillaris ischaemia could give rise to this illness, or instead, these vascular changes may emerge in response to an earlier lesion linked to senescence of the retinal pigment epithelium. ${ }^{15}$ The existence of an original vascular lesion is most clearly established in arteritic AION, in which massively delayed choroidal filling occurs as a consequence of posterior ciliary arterial occlusion. ${ }^{20}$ The vascular factors in the more common idiopathic non-arteritic AION are less clear, although delayed prelaminar filling of the oedematous disc in the acute stage of the illness supports the concept of an initial vascular lesion. ${ }^{21}$ The association of the disease with ischaemic heart disease, hypercholesterolaemia, diabetes and nocturnal hypotension also suggests that circulatory dysfunction may be involved. ${ }^{22,23}$ However, the precise roles that nocturnal hypotensive episodes, defective optic nerve head blood flow autoregulation, vascular endothelial dysfunction or small disc area may play in the aetiology of this illness have yet to be specified. ${ }^{21}$

In conclusion, ischaemia can provoke a diverse array of pathological changes in the central nervous system, the specific lesions apparently depending upon both the duration and the severity of the vascular insult, and upon the structure and cellular capacities of the tissues affected. While the critical experiments have yet to be performed, the possibility remains open that the distinctive pathologies of glaucoma, AMD and AION could all arise in toto or in part from compromised delivery of nutrients to ocular tissues. If this hypothesis is borne out, vasoprotection may yet prove to be a primary avenue for disease prevention across a wide range of ocular illnesses.

\section{References}

1. Chew SJ, Ritch R. Neuroprotection: the next breakthrough in glaucoma? Proceedings of the Third Annual Optic Nerve Rescue and Restoration Think Tank. J Glaucoma 1997;6:263-6.

2. Schielke GP, Yang GY, Shivers BD, et al. Reduced ischemic brain injury in interleukin-1 beta converting enzymedeficient mice. J Cereb Blood Flow Metab 1998;18:180-5.

3. Hayreh SS. Retinal and optic nerve head ischemic disorders and atherosclerosis: role of serotonin. Prog Ret Eye Res 1999;18:191-221.

4. Cummins D, Kitano S, Caprioli J. The effects of excitatory amino acids and ischemia on cultured rat retinal ganglion cells. Invest Ophthalmol Vis Sci 1992;33:1031-5.

5. Kitano S, Caprioli J. Expression of $72 \mathrm{kDa}$ heat shock protein in cultured rat retinal ganglion cells: ischemic tolerance and protective effect of hyperthermia. Invest Ophthalmol Vis Sci 1993;34:1430-4.

6. Koseki Y, Kitano S, Podos SM, et al. A nitric oxide synthase inhibitor protects against anoxia in cultured rat retinal ganglion cells in culture. Invest Ophthalmol Vis Sci 1994;335:1868-71.

7. Lin B, Ginsberg MD, Busto R, et al. Sequential analysis of subacute and chronic neuronal, astrocytic and microglial alterations after transient global ischemia in rats. Acta Neuropathol 1998;95:511-23.

8. Keller JN, Kindy MS, Holstberg FW, et al. Mitochondrial manganese superoxide dismutase prevents neural apoptosis and reduces ischemic brain injury: suppression of peroxynitrite production, lipid peroxidation, and mitochondrial dysfunction. J Neurosci 1998;18:687-97.

9. Li Y, Chopp M, Powers C, et al. Apoptosis and protein expression after focal cerebral ischemia in rat. Brain Res 1997;765:301-12.

10. Pennypacker KR, Hernandez H, Benkovic $\mathrm{S}$, et al. Induction of presenilins in the rat brain after middle cerebral arterial occlusion. Brain Res Bull 1999;48:539-43.

11. Kalaria RN, Cohen DL, Premkumar DR, et al. Vascular endothelial growth factor in Alzheimer's disease and experimental cerebral ischemia. Brain Res Mol Brain Res 1998;62:101-5

12. Burkart KK, Beard DC, Lehman RA, et al. Alterations in tau phosphorylation in rat and human neocortical brain slices following hypoxia and glucose deprivation. Exp Neurol 1998;154:464-72.

13. Shi J, Panickar KS, Yang SH, et al. Estrogen attenuates overexpression of beta-amyloid precursor protein messager RNA in an animal model of focal ischemia. Brain Res 1998;810:87-92.

14. Lin B, Schmidt-Kastner R, Busto R, et al. Progressive parenchymal deposition of beta-amyloid precursor protein in rat brain following global cerebral ischemia. Acta Neuropathol 1999;97:359-68.

15. Friedman E, Krupsky S, Lane A, et al. Ocular blood flow velocity in age-related macular degeneration. Ophthalmology 1995;102:640-6. 
16. Yin ZQ, Vaegan A, Millar TJ, et al. Widespread choroidal insufficiency in primary open-angle glaucoma. J Glaucoma 1997;6:23-32.

17. Rankin SJ, Walman BE, Buckley AR, et al. Color Doppler imaging and spectral analysis of the optic nerve vasculature in glaucoma. Am J Ophthalmol 1995;119:685-93.

18. Michelson G, Langhans MJ, Harazny J, et al. Visual field defect and perfusion of the juxtapapillary retina and the neuroretinal rim in primary open-angle glaucoma. Graefes Arch Clin Exp Ophthalmol 1998;236:80-5.

19. Remulla JF, Gaudio AR, Miller S, et al. Foveal electroretinograms and choroidal perfusion characteristics in fellow eyes of patients with unilateral neovascular agerelated macular degeneration. Br J Ophthalmol 1995;79:558-61.
20. Valmaggia C, Speiser $P$, Bischoff $P$, et al. Indocyanine green versus fluorescein angiography in the differential diagnosis of arteritic and nonarteritic anterior ischemic optic neuropathy. Retina 1999;19:131-4.

21. Lessell S. Nonarteritic anterior ischemic optic neuropathy: enigma variations. Arch Ophthalmol 1999;117:386-8.

22. Salomon O, Huna-Baron R, Kurtz S, et al. Analysis of prothrombotic and vascular risk factors in patients with nonarteritic anterior ischemic optic neuropathy. Ophthalmology 1999;106:739-42.

23. Hayreh SS, Podhajsky P, Zimmerman MB. Role of nocturnal arterial hypotension in optic nerve head ischemic disorders. Ophthalmologica 1999;213:76-96. 